\title{
Desempeño diagnóstico de los síntomas y signos de COVID-19 en pacientes adultos
}

\author{
Diagnostic performance of symptoms and signs of COVID-19 in adults
}

\section{Comentado de:}

Struyf T, et al. Cochrane Database Syst Rev. $2021 \mathrm{Feb}$ 23;2:CD013665. PMID: 33620086 ${ }^{1}$

\section{Antecedentes}

Las consecuencias clínicas de la infección por SARS-COV-2 son muy variables. El abanico clínico varía entre la infección asintomática hasta la afección crítica que requiere asistencia respiratoria mecánica, y, en algunos casos, alcanza la muerte, especialmente en los adultos mayores. Los síntomas como la fiebre, la tos, o la pérdida del olfato o el gusto, y los signos como la saturación de oxígeno, son la primera información de sospecha diagnóstica. Esa información podría utilizarse para descartar COVID-19, o para seleccionar a los pacientes que realizarán más pruebas. Esta es una actualización de la revisión cuya primera versión se publicó en julio de 2020.

\section{Objetivos}

Evaluar la exactitud diagnóstica de los signos y síntomas para determinar si una persona que acude a servicios de atención primaria o de urgencias, tiene COVID-19.

\section{Métodos de búsqueda}

Para esta actualización se realizaron búsquedas electrónicas hasta el 15 de julio de 2020 en el registro de estudios COVID-19 de Cochrane (Cochrane COVID-19 Study Register) y en la University of Bern living search database. Además, se examinaron los repositorios de publicaciones sobre COVID-19. No se aplicaron restricciones de idioma.

\section{Criterios de selección}

Los estudios fueron elegibles si incluían a pacientes con sospecha clínica de COVID-19 o si reclutaban casos confirmados de COVID-19 y controles sin COVID-19. Otra condición era que reclutaran a pacientes que acudían a servicios de atención primaria o ambulatorios de un hospital. Los estudios en pacientes hospitalizados sólo se incluyeron si se registraron los síntomas y signos en el momento del ingreso o en el momento de acudir al hospital. Los estudios que incluían a pacientes que contrajeron la infección por SARS-CoV-2 mientras estuvieron ingresados en el hospital no fueron elegibles. El tamaño mínimo de la muestra elegible fue de diez participantes. Todos los signos y síntomas fueron incluidos para esta revisión, incorporando signos y síntomas individuales o combinaciones. Se aceptaron varios patrones de referencia.

\section{Obtención y análisis de los datos}

Pares de autores de la revisión completaron de forma independiente la selección de los estudios, y cualquier desacuerdo se resolvió mediante discusión con un tercer autor. Dos autores de la revisión extrajeron los datos de forma independiente y resolvieron los desacuerdos mediante discusión con un tercer autor. El par de revisores también evaluaba de manera independiente el riesgo de sesgo con la lista de verificación Quality Assessment tool for Diagnostic Accuracy Studies (QUADAS-2). Se presentaron la sensibilidad y especificidad en diagramas de bosque (forest plots) emparejados, en el espacio de la curva de rendimiento diagnóstico y en diagramas de puntos conectados (dumbbell plots). Se calcularon los parámetros mediante un meta análisis bivariado de efectos aleatorios siempre que se dispusiera de al menos cinco estudios primarios, y siempre que la heterogeneidad entre los estudios se considerara aceptable.

\section{Resultados principales}

Se identificaron 44 estudios con 26.884 participantes en total. La prevalencia de COVID-19 varió del $3 \%$ al $71 \%$, con una mediana de $21 \%$. Tres estudios provinieron de ámbitos de atención primaria (1.824 participantes), nueve estudios de centros ambulatorios de pruebas para COVID-19 (10.717 participantes), 12 estudios se realizaron en servicios ambulatorios hospitalarios (5.061 participantes), siete estudios en pacientes hospitalizados (1.048 participantes), diez estudios en servicios de urgencias (3.173 participantes) y tres estudios no especificaron el contexto (5.061 participantes). Los estudios no distinguieron claramente la intensidad del cuadro clínico, por lo que se presentan juntos los resultados para todos los tipos de intensidad de la enfermedad.

En 15 estudios se identificó un alto riesgo de sesgo de selección de participantes porque la inclusión en los estudios dependía de los protocolos de prueba y de derivación aplicables, que incluían muchos de los signos y síntomas objeto de estudio en esta revisión. Esto podría haber influido especialmente en la sensibilidad de los aspectos utilizados en los protocolos de derivación, como la fiebre y la tos. Cinco estudios sólo incluyeron a participantes con neumonía en el diagnóstico por imágenes, lo que sugiere que se trata de una población altamente seleccionada. En otros 12 estudios, no fue posible evaluar el riesgo de sesgo de selección. Esto hace que sea muy difícil evaluar la validez de la exactitud diagnóstica de los signos y síntomas a partir de los estudios incluidos.

La aplicabilidad de los resultados de esta actualización de revisión mejoró en comparación con la revisión original. Un mayor porcentaje de estudios incluyó a participantes que acudieron a servicios ambulatorios, que es donde se realizan la mayoría de las evaluaciones clínicas para COVID-19. Sin embargo, aún así, ninguno de los estudios presentó datos de niños por separado y solo un estudio se centró específicamente en adultos mayores.

Se encontraron datos de 84 signos y síntomas. Los resultados fueron muy variables entre los estudios. La mayoría tenían una sensibilidad muy baja y una especificidad alta. Sólo la tos (25 estudios) y la fiebre (siete estudios) tuvieron una sensibilidad agrupada de al menos el $50 \%$, pero las especificidades fueron de moderadas a bajas. La tos tuvo una sensibilidad del 67,4\% (intervalo de confianza [IC] del $95 \%: 59,8 \%$ a $74,1 \%$ ) y una especificidad del $35,0 \%$ (IC $95 \%: 28,7 \%$ a $41,9 \%$ ). La fiebre tuvo una sensibilidad del 53,8\% (IC 95\%: 35,0\% a $71,7 \%$ ) y una especificidad del 67,4\% (IC 95\%: 53,3\% a 78,9\%). El cociente agrupado de probabilidad positiva de la tos fue de tan solo el 1,04 (IC 95\%: 0,97 a 1,11) y el de la fiebre del 1,65 (IC $95 \%: 1,41$ a 1,93).

La anosmia sola (11 estudios), la ageusia sola (seis estudios) y la anosmia o la ageusia (seis estudios) tuvieron sensibilidades inferiores al $50 \%$, pero especificidades superiores al $90 \%$. La anosmia tuvo una sensibilidad agrupada del $28,0 \%$ (IC $95 \%$ : $17,7 \%$ a $41,3 \%$ ) y una especificidad del $93,4 \%$ (IC $95 \%: 88,3 \%$ a $96,4 \%$ ). La ageusia tuvo una sensibilidad agrupada del $24,8 \%$ (IC $95 \%: 12,4 \%$ a $43,5 \%$ ) y una especificidad del $91,4 \%$ (IC $95 \%: 81,3 \%$ a $96,3 \%$ ). La anosmia o ageusia tuvo una sensibilidad agrupada del $41,0 \%$ (IC $95 \%: 27,0 \%$ a $56,6 \%$ ) y una 
especificidad del 90,5\% (IC 95\%: 81,2\% a 95,4\%). Los cocientes agrupados de probabilidad positiva de la anosmia sola y de la anosmia o la ageusia fueron 4,25 (IC 95\%: 3,17 a 5,71) y 4,31 (IC $95 \%: 3,00$ a 6,18$)$ respectivamente, lo que está justo por debajo de la definición arbitraria de "síntoma de alarma", es decir, un cociente de probabilidad positiva de al menos 5 . El cociente agrupado de probabilidad positiva de la ageusia sola fue de tan solo el 2,88 (IC 95\%: 2,02 a 4,09).

Solo dos estudios evaluaron combinaciones de diferentes signos y síntomas, principalmente combinando la tos y la fiebre con otros síntomas. Estas combinaciones tenían una especificidad superior al $80 \%$, pero a costa de una sensibilidad muy baja (menor al $30 \%$ ).

\section{Conclusiones de los autores}

La mayoría de los signos y síntomas individuales incluidos en esta revisión parecen tener una exactitud diagnóstica muy deficiente, aunque esto debe interpretarse en el contexto del sesgo de selección y la heterogeneidad entre los estudios. Sobre la base de los datos disponibles actualmente, ni la ausencia ni la presencia de signos o síntomas son lo suficientemente exactas como para confirmar o descartar COVID-19. La presencia de anosmia o ageusia podría ser útil como síntoma de alarma para COVID-19. La presencia de fiebre o tos, dadas sus altas sensibilidades, también podría ser útil para identificar a las personas para realizarles más pruebas.

Todavía se necesitan con urgencia estudios prospectivos en una población no seleccionada que acuda a los centros de atención primaria o a los servicios ambulatorios hospitalarios, que examinen combinaciones de signos y síntomas para evaluar el cuadro sindrómico inicial de COVID-19. Los resultados de esos estudios podrían servir de base para posteriores decisiones de gestión.

Fuente de financiamiento/Conflicto de interés de los autores: Esta revisión fue financiada por la Escuela de Medicina Tropical de Liverpool y la Universidad de Birmingham, Reino Unido, y por subsidios de investigación de entidades públicas y universitarias de ese país. La mayoría de los autores declararon no tener conflictos de interés, mientras que uno de ellos declaró ser empleado de una organización sin fines de lucro asociada a la Organización Mundial de la Salud denominada FIND, dedicada a acelerar el acceso a herramientas de diagnóstico de alta calidad para entornos de bajos recursos.

\section{Comentario}

En la actualidad la humanidad está viviendo una experiencia sanitaria y social única. La aparición a fines de 2019 de un nuevo agente etiológico, cuyo origen aún no está del todo esclarecido ${ }^{2}$, ha llevado a una pandemia con consecuencias sanitarias, culturales y económicas devastadoras en muchos países. A pesar de haber transcurrido 18 meses desde los primeros casos reportados en el mundo como COVID-19, todavía se está aprendiendo sobre esta enfermedad en todos sus aspectos.

A medida que se contagian más personas, las características clínicas de las distintas maneras de presentación de la posible enfermedad se diversifican, por diferentes motivos. Solo para mencionar algunos aspectos, sabemos que la clínica varía mucho con la edad del paciente afectado, sus comorbilidades previas y sus características socioeconómicas ${ }^{3}$. Ahora también se está estudiando si los diferentes genotipos del mismo virus que se han descubierto se asocian a patrones de presentación clínica particular ${ }^{4}$. Como ha pasado tantas veces en la historia de las enfermedades infecciosas, las características del agente etiológico, del huésped, y de su hábitat se combinan para manifestar las distintas formas de presentación, y así surgen las formas incipientes, las probables, las típicas, las atípicas, las de poblaciones especiales (como podrían ser las personas inmunosuprimidas, o con HIV), etc.

En esta revisión Cochrane, se documentó que los síntomas relacionados con más frecuencia con la COVID-19, como la tos y la fiebre, tienen valores bajos de sensibilidad, especificidad, y coeficiente de probabilidad positiva. Por su parte, la anosmia y la ageusia tienen mejor desempeño en su especificidad y coeficiente de probabilidad positiva, pero sin llegar a valores habitualmente considerados apropiados. Asimismo, se destaca el alto grado de incertidumbre acerca del valor diagnóstico de los síntomas y signos en forma individual, así como de sus combinaciones, que fueron poco estudiadas hasta el momento. Una de las limitaciones de esta revisión es haber abarcado a pacientes adultos, con poca representación de ancianos, y no haber incluido a niños ni a personas gestantes. Al mismo tiempo, tiene la fortaleza de sintetizar la evidencia disponible sobre la clínica de COVID-19 en el ámbito de la atención ambulatoria, en el que se desarrollan tantas batallas contra la enfermedad y que desde el inicio quedó retrasado en la investigación, basada principalmente en los pacientes internados ${ }^{5}$. Sin embargo, las principales limitaciones de esta revisión consisten en las debilidades metodológicas de los estudios primarios, con alto riesgo de sesgos de selección y de detección, y la sustancial heterogeneidad en los métodos de recolección de los datos (de qué manera, en qué momento y qué persona determinaba la presencia de los signos y síntomas).

Se podría esperar que la recolección masiva de datos clínicos de pacientes individuales como consecuencia de la aplicación de protocolos de manejo de casos sospechosos durante la pandemia permita realizar análisis más robustos del valor diagnóstico de diferentes combinaciones de síntomas y signos para esta enfermedad. En la región, un estudio reciente realizado en Chile con una muestra de participantes del estudio Monitoreo Nacional de Síntomas y Prácticas COVID-19 (MOVID-19) documentó las propiedades diagnósticas de los criterios de caso sospechoso de esta enfermedad empleados durante $2020^{6}$. Sin embargo, estos criterios incluyeron datos adicionales, como el antecedente de contacto estrecho con un caso sospechoso o confirmado, por lo que sus resultados no son comparables con los sintetizados en la revisión sistemática comentada.

\section{Conclusiones de los comentadores}

Los síntomas y signos tienen un desempeño diagnóstico (sensibilidad y especificidad) deficiente para COVID-19 cuando se los considera en forma aislada. Queda pendiente la evaluación de las diferentes combinaciones de síntomas y signos, que hasta el momento fueron poco estudiadas, a pesar de que se han acumulado datos observacionales a gran escala en los 
diferentes países en los que se emplean protocolos estrictos para determinar la realización de pruebas diagnósticas y las medidas de aislamiento tendientes a contener la propagación de esta enfermedad en la población.

\section{Diego Terceiro [ Servicio de Medicina Familiar y Comunitaria, Hospital Italiano de Buenos Aires. diego.terceiro@hospitalitaliano.org.ar ]}

Valeria Vietto [ Servicio de Medicina Familiar y Comunitaria, Hospital Italiano de Buenos Aires; Departamento de Investigación, Instituto Universitario Hospital Italiano de Buenos Aires. valeria.vietto@hospitalitaliano.org.ar ]

Terceiro D, Vietto V. Desempeño diagnóstico de los síntomas y signos de COVID-19 en pacientes adultos. Evid Actual Pract Ambul. 2021;24(2):e002129. Available from: https://dx.doi.org/10.51987/evidencia.v24i3.6935. Comentado de: Struyf T, et al. Signs and symptoms to determine if a patient presenting in primary care or hospital outpatient settings has COVID-19. Cochrane Database Syst Rev. 2021 Feb 23;2:CD013665. PMID: 33620086

\section{Referencias}

1. Struyf T, Deeks JJ, Dinnes J, et al. Signs and symptoms to determine if a patient presenting in primary care or hospital outpatient settings has COVID-19 disease. Cochrane Database Syst Rev. 2021;2:CD013665. Available from: 10.1002/14651858.CD013665.pub2.

2. La OMS pide más estudios y datos sobre el origen del SARS-CoV-2 y reitera que todas las hipótesis siguen abiertas; 2021 Available from: https://www.who.int/es/news/item/30-03-2021-who-calls-for-further-studies-data-on-origin-of-sars-cov-2-virus-reiterates-that-allhypotheses-remain-open [Last access: 2021-05-26].

3. Mclntosh K. Up To Date. In: Hirsch MS, Bloom A, editors. COVID-19: Clinical features. Wolters Kluwer; 2021

4. Frampton D, Rampling T, Cross A, et al. Genomic characteristics and clinical effect of the emergent SARS-CoV-2 B.1.1.7 lineage in London, UK: a whole-genome sequencing and hospital-based cohort study. Lancet Infect Dis. 2021;S1473-3099(21):00170-5. Available from: 10.1016/S14733099(21)00170-5.

5. Terceiro D, Vietto V. COVID-19: Presentación clínica en adultos. Evid Actual Pract Ambul. 2020;23(2):e002042. Available from: 10.51987/evidencia. v23i2.4290; http://www.evidencia.org.ar/index.php/Evidencia/article/view/4290.

6. Aubert J, Durán D, Monsalves MJ, et al. Propiedades diagnósticas de las definiciones de caso sospechoso de COVID-19 en Chile, 2020. Rev Panam Salud Publica. 2021;45:e14. Available from: 10.26633/RPSP.2021.14;https://iris.paho.org/handle/10665.2/53283. 